\title{
CONTRACTIBLE 3-MANIFOLDS OF FINITE GENUS AT INFINITY
}

\author{
BY
}

E. M. BROWN

\begin{abstract}
A class of contractible open 3-manifolds is defined. It is shown that all contractible open 3-manifolds which can be written as a union of cubes with a bounded number of handles are in this class. It is shown that a proper map between manifolds of this class which induces an isomorphism of proper fundamental groups (e.g. a proper homotopy equivalence) is proper homotopic to a homeomorphism. A naturality condition for homomorphisms of proper fundamental groups is developed. It is shown that a natural homomorphism between the proper fundamental groups of these manifolds is induced by a proper map.
\end{abstract}

1. Introduction and notation. In [12] J. H. C. Whitehead gave the first example of a contractible open 3-manifold not homeomorphic to $\mathbf{R}^{3}$ (see also [14]). Whitehead's example is an open subset of $\mathbf{R}^{3}$, so questions of the Poincaré conjecture do not arise and the phenomenon is a purely noncompact one. In [7] McMillan generalized Whitehead's construction to give an uncountable family of contractible open subsets of $\mathbf{R}^{3}$ with no two homeomorphic. Each member of McMillan's family is written as an increasing union of copies of a cube-with-one-handle, $S^{1} \times D^{2}$. It is not hard, following McMillan's construction, to give contractible open subsets of $\mathbf{R}^{3}$ that are unions of cubes with any number of handles. Indeed it follows from [8] or [9] that any contractible open 3-manifold with no fake 3-cells is a union of cubes-with-handles.

In [2] T. Tucker and this author generalized work of Waldhausen [11] and Heil [5] on compact 3-manifolds. We showed that for certain noncompact 3-manifolds one can reduce the question of homeomorphism to an algebraic question in proper homotopy. The theorems there do not apply, however, to contractible 3-manifolds.

In this paper we consider contractible open 3-manifolds which are irreducible and eventually end-irreducible (see below for the definition). Among these are all contractible open 3-manifolds which can be written as a union of cubes with a bounded number of handles. In $\$ 2$ we show that a proper map

Received by the editors November 11, 1977.

AMS (MOS) subject classifications (1970). Primary 57A10.

Key words and phrases. Proper map, proper homotopy equivalence, proper fundamental group, end, eventually end-irreducible, open 3-manifold.

๑) American Mathematical Society 1979 
between such manifolds which induces an isomorphism of the proper fundamental groups is proper homotopic to a homeomorphism. In particular this is true of any proper homotopy equivalence. This leads us to the question of whether each isomorphism of proper fundamental groups of 3-manifolds is induced by a proper map. Even for contractible open 3-manifolds this is false. In $\$ 3$ we develop a necessary and sufficient condition for a homomorphism between the proper fundamental groups of two contractible open 3-manifolds to be induced by a proper map. This gives an algebraic answer to the question of when two of the manifolds we are considering are homeomorphic.

In what follows we shall assume that all spaces are countable simplicial complexes and that all maps are piecewise linear. In general we follow the notation of [11]. An exception is that for us a manifold $M$ is an orientable 3-manifold which may be noncompact and which may have boundary, denoted $\partial M$. Another is that we use surface to mean a compact 2-manifold which may be disconnected and which may have boundary. Further, when we speak of a surface $F$ in a manifold $M$ we mean that $F$ is embedded in $M$ and that either $F \cap \partial M=\partial F$ or that $F$ consists of some components of the boundary of $M$. A manifold $M$ is irreducible if every 2-sphere in $M$ bounds a 3-cell. A surface $F$ in $M$ is compressible if some component of $F$ is a 2-sphere which bounds a 3-cell or if there is an embedded disk $D$ in $M$ so that $D \cap F=\partial D$ is an essential loop on $F$. Otherwise $F$ is said to be incompressible.

A map $f: X \rightarrow Y$ is proper if $f^{-1}(C)$ is compact for every compact $C \subset Y$. For notation in proper homotopy theory we follow [1] and [2]. An exception is an exhausting sequence for a 3-manifold $M$ is a sequence $\left\{M_{n}\right\}$ of compact submanifolds of $M$ so that $M_{n} \subset M_{n+1}-\partial\left(M_{n+1}\right)$ and $\cup M_{n}=M$. We use end-irreducible just as in [2], as an alternative the reader may use the conclusion of Lemma (2.1) as a definition. We say $M$ is eventually endirreducible if there is a compact submanifold $C$ of $M$ so that $\mathrm{cl}(M-C)$ is end-irreducible. As an illustration of these concepts, if $N$ is a compact manifold with $\partial N \neq \varnothing$, then $N-\partial N$ is eventually end-irreducible. If $\partial N$ is incompressible then $N-\partial N$ is end-irreducible. It is possible to construct a contractible open subset of $\mathbf{R}^{3}$ which is not eventually end-irreducible (see Figure 1).

Proper homotopy groups are defined in [1], we recall the definition of the proper fundamental group. First, proper maps $f$ and $g$ are equivalent, written $f=g$, if they agree on the complement of a compact set. $\underline{S}^{1}$ is the subspace of $\overline{\mathbf{R}}^{2}$ given in polar coordinates by $\underline{S}^{1}=\{(r, 0) \mid r>0\} \cup\{(n, \theta) \mid n=1$, $2, \ldots\}$. We use $\underline{*}=\{(r, 0) \mid r \geqslant 0\}$ as our analogue of the base point. For $n=1,2, \ldots$ the set $\{(n, \theta)\}$ is denoted $S^{1} \times\{n\}$. For $a: \neq \rightarrow K$ a proper map, the elements of the proper fundamental group $\underline{\pi}_{1}(K, \underline{a})$ are classes, $[\lambda]$, of proper maps $\lambda$ : $\underline{S}^{1} \rightarrow K$. We require $\lambda \mid \underline{*}$ to be equivalent to $a$. We define 
$[\lambda]=[\mu]$ provided there is proper homotopy $F: \underline{S}^{1} \times I \rightarrow K$ so that $F(x, t)=$ $\lambda(x)$ for $(x, t) \in(\underline{*} \times I) \cup\left(\underline{S}^{1} \times\{0\}\right)$, and $F(x, 1)$ is equivalent to $\mu$. For $[\lambda]$, $[\mu] \in \underline{\pi}_{1}(K, \underline{a})$ we construct a representative $\nu$ of $[\lambda] \cdot[\mu]$ by choosing $\lambda$ and $\mu$ to agree on $*$ and defining

$$
\nu(n, \theta)=\begin{array}{ll}
\lambda(n, 2 \theta), & 0 \leqslant \theta \leqslant \pi, \\
\mu(n, 2 \theta-\pi), & \pi \leqslant \theta \leqslant 2 \pi .
\end{array}
$$

The usual proofs in homotopy theory show the above multiplication is well defined, and that with this multiplication, $\underline{\pi}_{1}(K, \underline{a})$ is a group. If $f: K \rightarrow L$ is a proper map then, by composition, $f$ induces a homomorphism $f_{*}: \underline{\pi}_{1}(K, \underline{a}) \rightarrow$ $\underline{\pi}_{1}(L, f a)$. Higher order proper homotopy groups, and induced homomorphisms are defined similarly.

An unlabeled homomorphism between homotopy groups or proper homotopy groups of spaces will always be induced by inclusion. We suppress explicit mention of base points when the choice does not affect the statement being made. On occasion we write $\pi_{1}(F)$ where $F$ is a disconnected surface. In such a case the statement is meant to apply to the fundamental group of each component of $F$.

Proper maps $a, b: * \rightarrow K$ define the same end of $K$, written $[a]=[b]$, provided for any compact set $C \subset K, a(t)$ and $b(t)$ are in the same component of $K-C$ for all $t$ sufficiently large. The set of ends of $K$ is written $\underline{\pi}_{0}(K)$. If $C \subset K$ is a compact set, and if $A$ is a component of $K-C$, then $\{[a] \mid a(t) \in A$ for all $t$ sufficiently large $\}$ is declared an open set in $\underline{\pi}_{0}(K)$. This defines a topology on $\underline{\pi}_{0}(K)$ with which it is homeomorphic to a closed subset of the Cantor set. Higher order proper homotopy groups, and induced homomorphisms are defined similarly.

2. Homeomorphisms of contractible manifolds. In this section we define the genus at infinity of a manifold and investigate those manifolds for which this genus is finite. We give conditions under which a proper map between contractible open manifolds of finite genus at infinity is proper homotopic to a homeomorphism.

(2.1) LEMMA. Let $M$ be an open connected manifold. Then $M$ is end-irreducible iff there is an exhausting sequence $\left\{C_{n}\right\}$ for $M$ so that $C_{n}$ is connected and $\partial C_{n}$ is incompressible in $M$ for every $n$.

Proof. The "only if" part is a special case of (3.1) of [2]. We demonstrate the reverse implication. Let $\left\{C_{n}\right\}$ be an exhausting sequence with the given properties. Let [ $a$ ] be an end of $M$, and let $A_{n}$ be the component of $M-C_{n}$ which contains the end $[a]$. For any choice of base point $a_{n} \in A_{n}$ it follows that $\pi_{1}\left(A_{n}, a_{n}\right) \rightarrow \pi_{1}\left(M, a_{n}\right)$ is a monomorphism. Applying the functor $\mathscr{P}$ of [1], we have that $\mathscr{P}\left(\pi_{1}\left(A_{n}, a_{n}\right)\right) \rightarrow \mathscr{P}\left(\pi_{1}\left(M, a_{n}\right)\right)$ is a monomorphism. But 
there are natural isomorphisms $\mathscr{P}\left(\pi_{1}\left(A_{n}, a_{n}\right)\right) \cong \underline{\pi}_{1}(M, \underline{a})$ and $\mathscr{P}\left(\pi_{1}\left(M, a_{n}\right)\right)$ $\cong \pi_{1}(M, \underline{a})$ for appropriate choice of $\underline{a}$. This demonstrates the lemma.

Let $M$ be an open manifold. The genus of $M$ at infinity is the smallest integer $k$ so that there is an exhausting sequence $\left\{C_{n}\right\}$ for $M$ with the genus of each $\partial C_{n}$ equal to $k$. (The genus of an orientable surface is just the number of handles.) If no such $k$ exists we say that $M$ has infinite genus at infinity.

Each member of the uncountable family of contractible open subsets of $\mathbf{R}^{3}$ referred to in the introduction is a manifold of genus one at infinity. In Figure 1 we give the stage $C_{n} \subset C_{n+1}$ of the construction of a contractible open subset of $\mathbf{R}^{3}$. If $M$ is the open set so constructed it is not hard to see that $\operatorname{cl}\left(M-C_{n}\right)$ is not end-irreducible. It follows from (2.3) below that $M$ has infinite genus at infinity. If $N$ is a compact manifold and the genus of $\partial N$ is $k$, then $N-\partial N$ has genus $k$ at infinity.

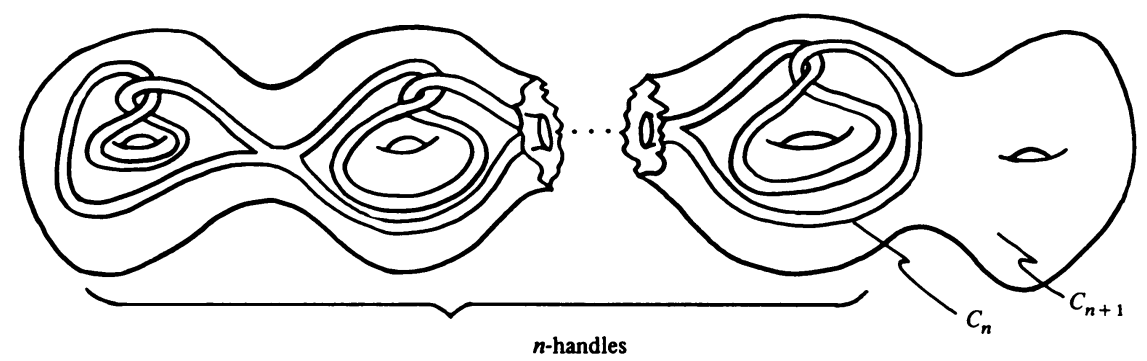

FIGURE 1

In what follows a bounded set is one with compact closure.

(2.2) LemMA. Let $M$ be a connected open manifold of genus $k>0$ at infinity having just one end. Suppose $H_{1}\left(M ; Z_{2}\right)$ is finitely generated. There exists an exhausting sequence $\left\{C_{n}\right\}$ for $M$ so that

(i) $\partial C_{n}$ has genus $k$,

(ii) $C_{n}, \partial C_{n}$, and $M-C_{n}$ are all connected,

(iii) $\partial C_{n}$ is incompressible in $\mathrm{cl}\left(M-C_{0}\right)$.

Proof. Let $\left\{C_{n}^{\prime}\right\}$ be an exhausting sequence for $M$ chosen so that the genus of $\partial C_{n}^{\prime}$ is $k$. By including in $C_{n}^{\prime}$ any bounded component of its complement, we may further assume that components of $M-C_{n}^{\prime}$ are unbounded. Then $M-C_{n}^{\prime}$ is connected. Let $C_{0}$ be any component of $C_{0}^{\prime}$ and let $C_{n}$ be the component of $C_{n}^{\prime}$ which contains $C_{0}$. It is not hard to see that $\left\{C_{n}\right\}$ is an exhausting sequence for $M$ and that $M-C_{n}$ is connected. By discarding a few initial terms if necessary, we may further assume that any compact submanifold containing $C_{0}$ has a boundary with genus at least $k$, and that $H_{1}\left(C_{0} ; Z_{2}\right) \rightarrow H_{1}\left(M ; Z_{2}\right)$ is an epimorphism. Since the genus of $\partial C_{n}$ is at most that of $\partial C_{n}^{\prime}$ we have that (i) is satisfied. 
We have chosen $C_{n}$ and $M-C_{n}$ connected, we claim $\partial C_{n}$ is also connected. If $\partial C_{n}$ is not connected let $F, G$ be components of $\partial C_{n}$. Let $p, q$ be points of $F, G$ respectively. Let $\lambda$ be the loop consisting of a path in $C_{n}$ joining $p$ to $q$, and a path in $\operatorname{cl}\left(M-C_{n}\right)$ joining $p$ to $q$. We regard $\lambda$ and $F$ as mod 2 cycles of $M$, their mod 2 intersection number is 1 . But if $\gamma$ is a mod 2 cycle of $C_{0}$, the mod 2 intersection number of $\gamma$ and $F$ is zero. This contradicts the fact that $H_{1}\left(C_{0} ; Z_{2}\right) \rightarrow H_{1}\left(M ; Z_{2}\right)$ is an epimorphism.

For (iii) let us suppose that $\partial C_{n}$ is compressible in $\operatorname{cl}\left(M-C_{0}\right)$ for some $n$. Let $D$ be an embedded disk in $\operatorname{cl}\left(M-C_{0}\right)$ with $D \cap \partial C_{n}=\partial D$ an essential loop on $\partial C_{n}$. Assume $D \cap C_{0}=\varnothing$ unless $n=0$. Let $U(D)$ be a regular neighborhood of $D$ in $C_{n}-C_{0}$ or $\operatorname{cl}\left(M-C_{n}\right)$, whichever contains $D$. Suppose, first, that $\partial D$ is a nonseparating loop in $\partial C_{n}$. If $D \subset C_{n}-C_{0}$ then $\operatorname{cl}\left(C_{n}-U(D)\right)$ contains $C_{0}$ but has boundary of genus less than $k$. This contradicts the choice of $C_{0}$. A similar contradiction arises if $D \subset \mathrm{cl}(M-$ $C_{n}$ ) by considering $C_{n} \cup U(D)$.

Now assume that $\partial D$ separates $\partial C_{n}$. If $D \subset C_{n}-C_{0}$ then $\operatorname{cl}\left(C_{n}-U(D)\right)$ has two boundary components each of positive genus less than $k$. Each bounds a compact submanifold of $M$, and one of these contains $C_{0}$. Again we have a contradiction, and one treats the case $D \subset \operatorname{cl}\left(M-C_{n}\right)$ as in the last paragraph. This establishes (iii) and the lemma.

(2.3) COROLLARY. If $M$ is a contractible open manifold of finite genus $k>0$ at infinity, then $M$ is eventually end-irreducible.

Proof. Since $M$ is contractible, (2.2) applies to $M$. Let $\left\{C_{n}\right\}$ be as in (2.2). Then by $(2.1), \operatorname{cl}\left(M-C_{0}\right)$ is end-irreducible.

The next theorem is analogous to Theorem (4.2) of [2], and is based on that theorem. It is a version of that theorem "off a compact set".

(2.4) THEOREM. Let $M$ and $N$ be connected, irreducible, open manifolds which are eventually end-irreducible and have one end each. Let $f: M \rightarrow N$ be a proper map which induces a monomorphism from $\underline{\pi}_{1}(M)$ to $\underline{\pi}_{1}(N)$. Then there exists a proper map $g: M \rightarrow N$ and a compact submanifold $\bar{D}$ of $N$ so that $g$ is proper homotopic to $f$ and $g \mid \operatorname{cl}\left(M-g^{-1}(D)\right)$ is a covering map onto $\operatorname{cl}(N-D)$. If $f$ induces an isomorphism then $g \mid \mathrm{cl}\left(M-g^{-1}(D)\right)$ is a homeomorphism.

Proof. Since $N$ has just one end we may choose an exhausting sequence $\left\{D_{n}\right\}$ for $N$ so that both $D_{n}$ and $N-D_{n}$ are connected. As $N$ is eventually end-irreducible we may further assume that $\operatorname{cl}\left(N-D_{0}\right)$ is end-irreducible and that $\partial D_{n}$ is incompressible in $\operatorname{cl}\left(N-D_{0}\right)$. Choose a compact manifold $C \subset M$ so that if $A=\operatorname{cl}(M-C)$ then $A$ is connected, end-irreducible, and $\partial A$ is incompressible in $A$. By a renumbering we may assume that $f(C) \subset D_{0}$ $-\partial D_{0}$.

There is a proper homotopy rel $\partial A$ of $f \mid A$ to a proper map $f^{\prime}: A \rightarrow N$ so 
that for every $n, f^{\prime}$ is transverse with respect to $\partial D_{n}$ and $f^{\prime-1}\left(\partial D_{n}\right)$ is incompressible in $A$ (cf. proof of (4.2) of [2]). We let $f^{\prime}|C=f| C$ and extend the proper homotopy to be constant on $C$. Then $f_{*}^{\prime}$ is a monomorphism or an isomorphism from $\underline{\pi}_{1}(M)$ to $\underline{\pi}_{1}(N)$ according as $f_{*}$ is a monomorphism or an isomorphism. Thus we may assume the above properties hold for $f$.

Let $A_{n}$ be the closure of the unbounded component of $M-f^{-1}\left(D_{n}\right)$. If $B_{n}=\operatorname{cl}\left(N-D_{n}\right)$ then $f_{n}=f \mid A_{n}$ is a proper map $f_{n}:\left(A_{n}, \partial A_{n}\right) \rightarrow\left(B_{n}, \partial B_{n}\right)$. We claim that $f_{n^{*}}: \pi_{1}\left(A_{n}\right) \rightarrow \pi_{1}\left(B_{n}\right)$ is a monomorphism for all sufficiently large $n$. To see this choose a base point $a(n) \in A_{n}$ for each $n$ and a path $a$ : $[n, n+1] \rightarrow A_{n}$ from $a(n)$ to $a(n+1)$. If $f_{n^{*}}$ is not a monomorphism we can choose an essential loop $\lambda_{n}$ in $A_{n}$ based at $a(n)$ so that $f_{n} \circ \lambda_{n}=f \circ \lambda_{n}$ is null-homotopic in $B_{n}$. Since $\partial C_{n}$ is incompressible in $A$ it follows that $\lambda_{n}$ is essential in $M-C$. If $f_{n^{*}}$ is a monomorphism we let $\lambda_{n}$ be the constant path at $a(n)$. The map $\lambda:\left(\underline{S}^{1}, \underline{*}\right) \rightarrow(M, \underline{a})$, defined by $\lambda \mid S^{1} \times\{n\}$ is the loop $\lambda_{n}$, is proper and represents an element of $\underline{\pi}_{1}(M, \underline{a})$. However $f_{*}([\lambda])=[f \circ \lambda]$ is the trivial element of $\underline{\pi}_{1}(N, \underline{f \circ a})$. Since $f_{*}$ is a monomorphism it follows that $\lambda_{n}$ is null-homotopic in $M-C$ for all sufficiently large $n$. By the above choice, $f_{n^{*}}$ is a monomorphism for all sufficiently large $n$.

We assume, by renumbering, that $f_{n^{*}}$ is a monomorphism for all $n$. We want to apply Theorem (4.2) of [2] to the proper map $f_{0}:\left(A_{0}, \partial A_{0}\right) \rightarrow$ $\left(B_{0}, \partial B_{0}\right)$. Note that $A_{0}$ and $B_{0}$ are connected, boundary irreducible, end-irreducible, and irreducible 3-manifolds. Moreover $\partial A_{0}$ and $\partial B_{0}$ are compact and $f_{0^{*}}$ is a monomorphism. Thus (4.2) of [2] applies and we have two possible conclusions denoted (a) and (b). Conclusion (b) requires that $\partial A_{0}=\varnothing$, which is false, thus we have conclusion (a): $f_{0}$ is proper homotopic as a map of pairs to a finite sheeted covering map $g_{0}: A_{0} \rightarrow B_{0}$. We extend this homotopy to a proper homotopy of $f$ to a proper map $g: M \rightarrow N$ so that $g \mid A_{0}=g_{0}$. Now $C_{0}=\operatorname{cl}\left(M-A_{0}\right)$ is compact and $g$ is proper, thus it follows that $g^{-1}\left(B_{m}\right) \subset$ $A_{0}$ for all $m$ sufficiently large. For such an $m, g \mid g^{-1}\left(B_{m}\right)$ is a covering map onto $B_{m}$ and we may choose $D=D_{m}$ to prove the theorem. Notice that as $M$ has only one end, $g^{-1}\left(B_{m}\right)$ is connected, that is, $g^{-1}\left(B_{m}\right)=A_{m}$.

Suppose now that $f_{*}$ is an isomorphism. Then so is $g_{*}$. Let $A_{n}=g^{-1}\left(B_{n}\right)$ and let $g_{n}=g \mid A_{n}$. If we can show that $g_{n^{*}}: \pi_{1}\left(A_{n}\right) \rightarrow \pi_{1}\left(B_{n}\right)$ is an epimorphism for some arbitrarily large values of $n$, then for such $n$ the covering of the preceding paragraph will be single sheeted. Suppose to the contrary that there exist arbitrarily large values of $n$ for which $g_{n^{*}}$ is not an epimorphism. For such values of $n$ pick $\left[\mu_{n}\right] \in \pi_{1}\left(B_{n}, g(a(n))\right)$ not in the image of $g_{n^{*}}$. For other values of $n$ let $\mu_{n}$ be the constant map to $g(a(n))$. Now define $\mu:\left(\underline{S}^{1}, \pm\right) \rightarrow(N, \underline{g \circ a})$ by $\mu \mid S^{1} \times\{n\}=\mu_{n}$. The map $\mu$ is proper and represents an element $[\mu] \in \underline{\pi}_{1}(N, g a)$. By hypothesis $[\mu]=g_{*}[\lambda]$ for some $[\lambda] \in \underline{\pi}_{1}(M, \underline{a})$. Let $\lambda_{n}=\lambda \mid S^{1} \times\{n\}$. Fix $m$, then for all sufficiently large $n$, $\lambda_{n}$ is a loop in $A_{m}$ and $g \circ \lambda_{n}$ is homotopic rel base point to $\mu_{n}$ in $B_{m}$. As $g \mid A_{m}$ 
is a covering map to $B_{m}$ we may cover the above homotopy to get a loop $\lambda_{n}^{\prime}$ in $A_{n}$ with $g \lambda_{n}^{\prime}=\mu_{n}$ for all sufficiently large $n$. Then if $\lambda^{\prime}:\left(\underline{S}^{1}, \underline{\underline{ }}\right) \rightarrow(M, \underline{a})$ is given by $\lambda^{\prime} \mid S^{1} \times[n]=\lambda_{n}^{\prime}, \lambda^{\prime}$ is a proper map and $[\mu]=\left[g \lambda^{\prime}\right]$. This contradicts the choice of $\mu_{n}$ and proves the theorem.

(2.5) THEOREM. Let $M$ and $N$ be contractible open manifolds which are irreducible and eventually end-irreducible. Let $f: M \rightarrow N$ be a proper map which induces an isomorphism $f_{*}: \underline{\pi}_{1}(M) \rightarrow \underline{\pi}_{1}(N)$. Then $f$ is proper homotopic to a homeomorphism.

Proof. It follows from Theorem 1 of [8] that each of $M$ and $N$ has an exhausting sequence of cubes-with-handles. Alternatively one may use Theorem 1 of [9] to reach the same conclusion. In particular $M$ and $N$ have one end each, and are connected. Let $\left\{C_{n}\right\}$ be an exhausting sequence of cubes-with-handles for $\boldsymbol{M}$.

By the above, Theorem (2.4) applies to $f$. Let $g: M \rightarrow N$, and $D \subset N$ be the map and the compact set of (2.4). As $g$ is a proper map, $g^{-1}(D) \subset C_{k}-\partial C_{k}$ for some $k$. The map $g \mid \partial C_{k}$ is a homeomorphism onto $g\left(\partial C_{k}\right)$. As $H_{2}\left(N ; Z_{2}\right)$ $=0, g\left(\partial C_{k}\right)$ is the boundary of a compact submanifold $C$ of $N$. We shall show that $g:\left(C_{k}, \partial C_{k}\right) \rightarrow(C, \partial C)$ is homotopic rel $\partial C_{k}$ to a homeomorphism, from which fact the theorem follows.

Suppose $C_{k}$ has $r$ handles. Let $D_{1}, \ldots, D_{r}$ be a set of disjoint disks in $C_{k}$ which cut all the handles. Then $g\left(\partial D_{1}\right), \ldots, g\left(\partial D_{r}\right)$ is a set of nonseparating disjoint simple loops in $\partial C$, each null-homotopic in $C$. By Dehn's lemma $g\left(\partial D_{i}\right)$ bounds an embedded disk $D_{i}^{\prime}$ in $C$, and by cut and paste arguments we may choose these disks disjoint. Since $N$ is irreducible, the result of cutting $C$ over $D_{1}^{\prime}, \ldots, D_{r}^{\prime}$ is a cell. By (1.3) of [11] there is a homotopy of $g \mid C_{k}$ rel $\partial C_{k}$ to a map $g^{\prime}: C_{k} \rightarrow C$ so that $g^{\prime-1}\left(\cup_{i} D_{i}^{\prime}\right)$ is a set of disks which cut $C_{k}$ into a cell, and possibly some closed incompressible surfaces. Clearly there are no closed incompressible surfaces in $g^{\prime-1}\left(\cup_{i} D_{i}^{\prime}\right)$, and it quickly follows that $g^{\prime}$ is homotopic rel $\partial C_{k}$ to a homeomorphism onto $C$.

Putting together (2.3) and (2.5) we get a characterization of certain contractible open manifolds. This applies to most of the examples hitherto constructed (the example of Figure 1 is an exception). It should be noted that a contractible open manifold will be irreducible if the terms of an exhausting sequence contain no fake 3-cells.

(2.6) Corollary. Suppose $M$ and $N$ are irreducible contractible open manifolds of finite genus at infinity. Suppose $f: M \rightarrow N$ is a proper map which induces an isomorphism $f_{*}: \underline{\pi}_{1}(M) \rightarrow \underline{\pi}_{1}(N)$ (e.g. $f$ is a proper homotopy equivalence). Then $f$ is proper homotopic to a homeomorphism of $M$ onto $N$.

3. The existence of proper maps. The results (2.5) and (2.6) of the last section require the existence of a proper map which induces an isomorphism 
of proper fundamental groups. In this section we show that the isomorphisms satisfying a certain naturality condition are exactly those induced by proper maps.

Let $K$ be a complex and let $a: \xi \rightarrow K$ be a proper map. Then composition of maps induces a function $c: \underline{\pi}_{1}\left(\underline{S}^{1}, \underline{*}\right) \times \underline{\pi}_{1}(K, \underline{a}) \rightarrow \underline{\pi}_{1}(K, \underline{a})$. If $L$ is another complex and $f: K \rightarrow L$ is proper, then the following diagram commutes:

$$
\begin{array}{ccc}
\underline{\pi}_{1}\left(\underline{S}^{1}, \underline{*}\right) \times \underline{\pi}_{1}(K, \underline{a}) & \stackrel{c}{\rightarrow} & \underline{\pi}_{1}(K, \underline{a}) \\
\downarrow \text { id } \times f_{*} & & \downarrow f_{*} \\
\underline{\pi}_{1}\left(\underline{S}^{1}, \underline{*}\right) \times \underline{\pi}_{1}(L, \underline{f a}) & \stackrel{c}{\rightarrow} & \underline{\pi}_{1}(L, \underline{f a})
\end{array}
$$

One can construct a complex $K$ and an isomorphism $\underline{\pi}_{1}(K, \underline{a}) \rightarrow \underline{\pi}_{1}(K, \underline{a})$ for which the above diagram does not commute. Thus commutativity is a necessary and nontrivial consequence of the fact that $f_{*}$ is induced by a proper map.

The following theorem needs a special condition on the map $a: * \rightarrow K$ which we later show can be relaxed for manifolds. The proof of the theorem is broken by Lemma (3.2) which has been used by Chipman [3], [4].

(3.1) TheOREM. Let $K$ be a finite dimensional complex. Let $a$ : $\sharp \rightarrow K$ be a proper map so that $a \mid[n, \infty)$ is a simplicial embedding for $n$ sufficiently large. Assume that the end $[a]$ of $K$ is isolated. Let $L$ be a contractible complex with an end $[b]$ so that $\underline{\pi}_{k}(L, \underline{b})=0$ for $1<k<\operatorname{dim} K$. Finally let $h: \underline{\pi}_{1}(K, \underline{a}) \rightarrow$ $\underline{\pi}_{1}(L, \underline{b})$ be a homomorphism so that the diagram

$$
\begin{array}{ccc}
\underline{\pi}_{1}\left(\underline{S}^{1}, \underline{*}\right) \times \underline{\pi}_{1}(K, \underline{a}) & \stackrel{c}{\rightarrow} & \underline{\pi}_{1}(K, \underline{a}) \\
\downarrow \text { id } \times h & & \downarrow h \\
\underline{\pi}_{1}\left(\underline{S}^{1}, \underline{*}\right) \times \underline{\pi}_{1}(L, \underline{b}) & \stackrel{c}{\rightarrow} & \underline{\pi}_{1}(L, \underline{b})
\end{array}
$$

commutes. Then there exists a proper map $f: K \rightarrow L$ so that $\underline{f a}=\underline{b}$ and $f_{*}=h$.

Proof. Choose a finite subcomplex $C \subset K$ so that:

(i) Components of $K-C$ are unbounded and

(ii) for some component $A$ of $K-C,[a]$ is the only end of $\operatorname{cl}(A)$.

Without changing $\underline{a}$ we may assume that for some integer $n$;

(iii) $a \mid[n, \infty)$ is a simplicial embedding,

(iv) $a \mid[0, n]$ is constant, and

(v) $a(\underline{*}) \cap C=a(0)=a([0, n])$.

As in the proof of Theorem (4.2) of [2], we can extend the tree $a(*)$ to a 
maximal tree $T$ of $\operatorname{cl}(A)$ so that $T$ has just one end. Notice that components of $T-a(\underline{*})$ are bounded.

Denote the 1-skeleton of $K$ by $K^{1}$. We define a proper map $\mu:\left(\underline{S}^{1}, \underline{*}\right) \rightarrow$ $\left(K^{1}, \underline{a}\right)$. Let $\left\{\sigma_{k}\right\}$ be a numbering of the 1-simplexes of $\operatorname{cl}(A)-T$, each chosen with an orientation. Let $\mu$ map $S^{1} \times\{k\}$ into $K^{1}$ as a loop which starts at $a(k)$ runs through $T$ directly to the initial vertex of $\sigma_{k}$, traverses $\sigma_{k}$ in the direction of its orientation, then returns through $T$ directly to $a(k)$. Let $\left.\mu\right|_{\underline{*}}=a$, then $\mu$ is a proper map.

(3.2) LEMMA. $\mu_{*}: \underline{\pi}_{1}\left(\underline{S}^{1}, \underline{*}\right) \rightarrow \underline{\pi}_{1}\left(K^{1}, \underline{a}\right)$ is an epimorphism.

Proof. Let $[\lambda] \in \underline{\pi}_{1}\left(K^{1}, \underline{a}\right)$. Assume the representative $\lambda:\left(\underline{S}^{1}, \underline{*}\right) \rightarrow\left(K^{1}, \underline{a}\right)$ of $[\lambda]$ is simplicial on a subdivision of $\underline{S}^{1}$, and $\lambda \mid \underline{\underline{*}}=a$. Let $\sigma_{m_{1}}^{\varepsilon_{1}}, \ldots, \sigma_{m_{t}}^{\varepsilon_{r}}$ be the sequence of 1-simplexes of $\operatorname{cl}(A)-T$ traversed as we run over the loop $\lambda \mid S^{1} \times\{m\}$. Here $\varepsilon_{i}=+1$ if $\lambda \mid S^{1} \times\{m\}$ runs over $\sigma_{m_{i}}$ in the positive direction, and $\varepsilon_{i}=-1$ otherwise. Let $\delta$ be the proper map of $\underline{S}^{1}$ to itself which is the identity on $*$ and which maps $S^{1} \times\{m\}$ around the loops $S^{1} \times\left\{m_{1}\right\}, \ldots, S^{1} \times\left\{m_{r}\right\}$. Here $\delta$ traverses $S^{1} \times\left\{m_{i}\right\}$ in the positive direction iff $\varepsilon_{i}=+1$. One easily checks that $\mu_{*}([\delta])=[\lambda]$.

Returning now to the hypotheses of (3.1), suppose $\mu$ is as defined above. Let $[\nu]=h([\mu]) \in \underline{\pi}_{1}(L, \underline{b})$. We claim there is a proper map $f: K \rightarrow L$ so that $\underline{f a}=\underline{b}$ and $f_{*}([\mu])=[\nu]$. If this is true then $f$ is the map promised in the statement of the theorem. We must only check that $f_{*}=h$. But for any $[\xi] \in \underline{\pi}_{1}(K, \underline{a})$ we have $[\xi]=\mu_{*}([\delta])$ some $[\delta] \in \underline{\pi}_{1}\left(\underline{S}^{1}, \underline{*}\right)$. Then $f_{*}([\xi])=$ $f_{*} \mu_{*}([\delta])=[f \circ \mu \circ \delta]=[\nu \circ \delta]=c([\delta],[\nu])=c([\delta], h([\mu]))=h(c([\delta],[\mu]))$ $=h([\xi])$.

To construct $f$, let $b: \underline{*} \rightarrow L$ be chosen so that $b \mid[0, n]$ is constant. Let $r: T \rightarrow a(\underline{*})$ be the proper retraction which maps each component $T_{i}$ of $T-a(*)$ to the single point $\operatorname{cl}\left(T_{i}\right) \cap a(*)$. Define $f$ on $T$ by $b \circ a^{-1} \circ r$. This is well defined since $a \mid[n, \infty]$ is an embedding while $b \mid[0, n]$ is constant. Moreover $T$ can be chosen so that on $T \cap C, f$ is the constant map to $b(0)$.

Now choose $m_{0}$ so that if $m \geqslant m_{0}$, then $\sigma_{m} \cap C=\varnothing$. Choose a representative $\nu:\left(\underline{S}^{1}, \underline{*}\right) \rightarrow(L, \underline{b})$ of $[\nu]$ so that $\nu \mid \underline{z}=b$, and $\nu \mid S^{1} \times\{m\}$ is constant for $m<m_{0}$. Proceeding as in the proof of (3.2) we can extend $f$ to a proper map of the 1-skeleton of $\operatorname{cl}(A)$ into $L$ so that $[f \circ \mu]=[\nu]$. Notice we may choose $f$ constant on $\sigma_{m}$ for $m<m_{0}$. If we can extend $f$ to a proper map of $K$ into $L$ then our claim is established.

We extend $f$ over $C$ to be the constant map to $b(0)$. This is consistent with our construction to this point. If $B$ is a component of $K-C$ other than $A$, then there is a proper map of $\operatorname{cl}(B)$ into $*$ which carries $\operatorname{cl}(B) \cap C$ into 0 . Indeed we get such a map by using the linear structure of successive closed stars of $\operatorname{cl}(B) \cap C$ in $\operatorname{cl}(B)$. Following such a map by $b: * \rightarrow L$ we extend $f$ properly over $K-A$ (any other proper extension would do as well). We shall 
be finished as soon as we extend $f$ properly over $A$.

Since $L$ is contractible, $f$ can be extended continuously over $K$ simplex by simplex, in order of increasing dimension. We let $\left\{D_{i}\right\}$ be an exhausting sequence for $L$, and we choose the extension of $f$ over each simplex $\sigma$ so that $f(\sigma) \cap D_{i}=\varnothing$ for $i$ as large as possible. We prove that $f$ is proper by induction on the skeletons $K^{r}$ of $K$. For $r=1, f$ is proper by construction. The case $r=2$ is special.

Suppose $f \mid K^{2}$ is not proper. Then there is a sequence $\left\{\sigma_{k}^{2}\right\}$ of distinct 2-simplexes of $A$, and an integer $i_{0}$, so that $f\left(\sigma_{k}^{2}\right) \cap D_{i_{0}} \neq \varnothing$. For each $k$ choose a path in $T$ from $a(k)$ to a base point on $\partial \sigma_{k}^{2}$. Choosing also an orientation of $\partial \sigma_{k}^{2}$ we define a proper map $\xi:\left(\underline{S}^{1}, \underline{*}\right) \rightarrow\left(K^{1}, \underline{a}\right)$ so that $[\xi]$ is the trivial element of $\underline{\pi}_{1}(K, \underline{a})$. However $[f \circ \xi]$ is not trivial in $\underline{\pi}_{1}(L, \underline{b})$ since $f\left(\partial \sigma_{k}^{2}\right)$ is essential in $\bar{L}-D_{i_{0}}$ for all $k$ sufficiently large. By (3.2) there is a proper map $\delta:\left(\underline{S}^{1}, \underline{*}\right) \rightarrow\left(\underline{S}^{1}, \underline{*}\right)$ so that $[\mu \circ \delta]=[\xi]$ in $\underline{\pi}_{1}\left(K^{1}, \underline{a}\right)$. Then $[f \circ \xi]=[f \circ \mu \circ \delta]=[\nu \circ \delta]=c(\delta, \nu)$ in $\underline{\pi}_{1}(L, \underline{b})$. However by the commutativity hypothesis we have $c(\delta, \nu)=\bar{c}(\delta, h[\mu])=h c(\delta, \mu)=h([\xi])=1$, where here $[\xi]$ and $\mu_{*}[\delta]$ means classes in $\underline{\pi}_{1}(K, \underline{a})$. This contradiction proves that $f \mid K^{2}$ is a proper map.

Finally suppose inductively that for some $r \geqslant 3$ we have shown $f \mid K^{r-1}$ is proper. If $f \mid K^{r}$ is not proper there is a sequence $\left\{\sigma_{k}^{r}\right\}$ of $r$-simplexes of $K$ converging to the end $[a]$ and an integer $i_{0}$ so that $f\left(\sigma_{k}^{r}\right) \cap D_{i_{0}} \neq \varnothing$. As above we use the sequence $\left\{\partial \sigma_{k}^{r}\right\}$ to define a proper map $\xi:\left(\underline{S}^{r-1}, \underline{*}\right) \rightarrow(K, \underline{a})$ so that $[\xi]=0$ in $\underline{\pi}_{r-1}(K, \underline{a})$ but $[f \circ \xi] \neq 0$ in $\pi_{r-1}(L, \underline{b})$. Since $r-1<\operatorname{dim} K$, we have $\pi_{r-1}(L, \underline{b})=\{0\}$, which is again a contradiction. Thus $f$ is constructed and the proof of (3.1) is finished.

Theorem (3.1) depended on special properties of the map $a: t \rightarrow K$ used as the "base point" for the proper fundamental group. To get a theorem about manifolds which does not depend on this special choice, we discuss the effect of "changing the base point" for the proper fundamental group.

Let $a_{0}, a_{1}: * \rightarrow K$ be proper maps, both representing the end $[a]$ of $K$. Choose a sequence of maps $p_{n}:[0,1] \rightarrow K$ so that $p_{n}(i)=a_{i}(n)$ for $i=0,1$, and so that the sets $p_{n}([0,1])$ converge to $[a]$ (converge to $[a]$ is defined in [2], it means the obvious thing). If $\beta:\left(\underline{S}^{1}, \underline{*}\right) \rightarrow\left(K, \underline{a}_{1}\right)$ is a proper map, we use the sequence $\left\{p_{n}\right\}$ to associate to $\bar{\beta}$ a proper map $\gamma:\left(\underline{S}^{1}, \underline{*}\right) \rightarrow\left(K, \underline{a}_{0}\right)$. If $\beta_{n}=\beta \mid S^{1} \times\{n\}$, then $\gamma_{n}=\gamma \mid S^{1} \times\{n\}$ is a standard representative of the change of base point of $\beta_{n}$ along $p_{n}$ to $a_{0}(n)$. The element $[\gamma] \in \underline{\pi}_{1}\left(K, \underline{a}_{0}\right)$ depends only on $[\beta] \in \underline{\pi}_{1}\left(K, \underline{a}_{1}\right)$ and on the proper homotopy class rel end points of the sequence $\left\{p_{n}\right\}$. Let $p_{*}([\beta])=[\gamma]$, it is not hard to check that $p_{*}: \underline{\pi}_{1}\left(K, \underline{a}_{1}\right) \rightarrow \underline{\pi}_{1}\left(K, \underline{a}_{0}\right)$ is an isomorphism. There is an obvious sense in which $p_{*}$ commutes with induced homomorphisms; however, it does not commute with the function $c$ defined at the beginning of this section.

Given $\left\{p_{n}\right\}$ we define a proper $\operatorname{map} \lambda:\left(\underline{S}^{1}, \underline{*}\right) \rightarrow\left(K, \underline{a}_{0}\right)$ 


$$
\lambda(n, \theta)= \begin{cases}p_{n}(2 \theta / \pi), & 0<\theta<\pi / 2, \\ a_{1}((2 \theta / \pi)+n-1,0), & \pi / 2<\theta<\pi, \\ p_{n+1}(-(2 \theta / \pi)+3), & \pi<0<3 \pi / 2, \\ a_{0}(-(2 \theta / \pi)+n+4,0), & 3 \pi / 2<\theta<2 \pi .\end{cases}
$$

If $[\lambda]$ is trivial in $\underline{\pi}_{1}\left(K, \underline{a}_{0}\right)$, then $p_{*}$ does commute with $c$. For example, let $\delta:\left(\underline{S}^{1}, \underline{*}\right) \rightarrow\left(\underline{S}^{1}, \underline{*}\right)$ wrap $S^{1} \times\{n\}$ once around $S^{1} \times\{n+1\}$. For any $[\mu] \in \underline{\pi}_{1}\left(K, \underline{a}_{1}\right)$ we have

$$
p_{*} c([\delta],[\mu])=[\lambda] \cdot c\left([\delta], p_{*}[\mu]\right) \cdot[\lambda]^{-1} \text {. }
$$

If a sequence $\left\{p_{n}\right\}$ exists for which $[\lambda]$ is trivial then there is a proper homotopy between $a_{0}$ and $a_{1}$. Conversely, if $H: * \times I \rightarrow K$ is a proper homotopy from $a_{0}$ to $a_{1}$, then $p_{n}(t)=H(n, t)$ is a sequence of paths for which $[\lambda]$ is trivial. We say that $p_{*}$ is induced by $H$ in this case. To conclude, if $p_{*}$ is induced by $H$, then $p_{*}$ commutes with $c$.

Suppose $K$ is a manifold and $a_{0}: * \rightarrow K$ is any given proper map. Using general position we can find a map $a_{1}: * \rightarrow K$ which is properly homotopic to $a_{0}$ and which is a simplicial embedding after subdivision. Using the above observations (3.1) can be improved, when $K$ is a manifold, by allowing $a$ to be any proper map.

(3.3) THEOREM. Let $M$ be a manifold, let $a: \pm \rightarrow M$ be a proper map, and assume the end $[a]$ of $M$ is isolated. Let $L$ be a contractible noncompact complex. Let $b^{\prime}:{ }_{-} \rightarrow L$ be a proper map and assume that $\underline{\pi}_{2}\left(L, \underline{b}^{\prime}\right)=\{0\}$. Suppose given a homomorphism $h: \underline{\pi}_{1}(M, \underline{a}) \rightarrow \underline{\pi}_{1}\left(L, \underline{b}^{\prime}\right)$ which commutes with c. Then there exists a proper map $f: \bar{M} \rightarrow L$ so that:

(i) There is a proper homotopy $H^{\prime}$ between $f a=b$ and $b^{\prime}$, and

(ii) if $p_{*}^{\prime}: \underline{\pi}_{1}(L, \underline{b}) \rightarrow \underline{\pi}_{1}\left(L, \underline{b}^{\prime}\right)$ is induced by $H^{\prime}$ then $p_{*}^{\prime} f_{*}=h$.

Proof. Choose a proper homotopy $H$ between $\underline{a}$ and a simplicial embed$\operatorname{ding} a^{\prime}: \underline{*} \rightarrow M$. Let $p_{*}: \underline{\pi}_{1}(M, \underline{a}) \rightarrow \underline{\pi}_{1}\left(M, \underline{a}^{\prime}\right)$ be the isomorphism induced by $H$. Let $h^{\prime}=h p_{*}^{-1}$ and note that $h^{\prime}$ also commutes with $c$. By (3.1) there is a proper map $f: M \rightarrow L$ so that $f a^{\prime}=b^{\prime}$ and $f_{*}=h^{\prime}$.

Let $H^{\prime}=f H$, this is a proper homotopy between $b=f a$ and $b^{\prime}=f a^{\prime}$. Let $p_{*}^{\prime}$ be the isomorphism induced by $H^{\prime}$. Then clearly $p_{*}^{\prime} f_{*}=f_{*} p_{*}$. But $f_{*} p_{*}=$ $h^{\prime} p_{*}=h$, and the theorem is proved.

(3.4) THEOREM. Let $M$ and $N$ be irreducible contractible open manifolds which are eventually end-irreducible. Let $h: \underline{\pi}_{1}(M) \rightarrow \underline{\pi}_{1}(N)$ be an isomorphism so that the diagram

$$
\begin{array}{ccc}
\underline{\pi}_{1}\left(\underline{S}^{1}\right) \times \underline{\pi}_{1}(M) & \stackrel{c}{\rightarrow} & \underline{\pi}_{1}(M) \\
\downarrow \text { id } \times h & & \downarrow h \\
\underline{\pi}_{1}\left(\underline{S}^{1}\right) \times \underline{\pi}_{1}(N) & \stackrel{c}{\rightarrow} & \underline{\pi}_{1}(N)
\end{array}
$$


commutes. Then there is a homeomorphism $f$ of $M$ onto $N$. Moreover $f_{*}$ and $h$ differ only by a change of base point isomorphism.

Proof. By (2.5) and (3.3) it is sufficient that $\underline{\pi}_{2}(N)=\{0\}$. Suppose not, and let $\left\{C_{n}\right\}$ be an exhausting sequence for $N$ with $N-C_{n}$ connected. It follows that for infinitely many values of $n, \pi_{2}\left(N-C_{n}\right) \neq 0$. Since $N$ is orientable we can apply the sphere theorem, [13] to $N-C_{n}$. This produces an embedded 2-sphere $\Sigma_{n} \subset N-C_{n}$, which represents a nonzero element of $\pi_{2}\left(N-C_{n}\right)$. Choosing a subsequence we may assume that $\Sigma_{n} \subset C_{n+1}-$ $\partial C_{n+1}$. Since $N$ is irreducible, $\Sigma_{n}$ bounds a 3-cell $B_{n} \subset N$. Now $B_{n}$ is not contained in $N-C_{n}$ since $\Sigma_{n}$ is essential there. It follows that $C_{n} \cap B_{n} \neq \varnothing$, and since $\Sigma_{n} \cap \partial C_{n}=\varnothing$, that $C_{n} \subset B_{n}$. Furthermore, $\Sigma_{n} \cap \partial C_{n+1}=\varnothing$ so $B_{n} \subset C_{n+1}-\partial C_{n+1}$. Thus $B_{n} \subset B_{n+1}-\partial B_{n+1}, \cup_{n} B_{n}=N$, and so $N$ is homeomorphic to $\mathbf{R}^{3}$. Then $\underline{\pi}_{1}(N)=\{1\}$, so $\underline{\pi}_{1}(M)=\{1\}$. We have that $M$ is homeomorphic to $\mathbf{R}^{3}$ as well, $h$ is the trivial homomorphism, and any homeomorphism will do for $f$.

\section{REFERENCES}

1. E. M. Brown, On proper homotopy type, Lecture Notes in Math., vol. 375, Springer-Verlag, Berlin and New York, 1973.

2. E. M. Brown and T. W. Tucker, On proper homotopy theory for noncompact 3-manifolds, Trans. Amer. Math. Soc. 188 (1974), 105-126.

3. J. C. Chipman, An isomorphism condition for towers of graphs, Pacific J. Math. (to appear).

4. _ Presentations for proper fundamental groups, preprint.

5. W. Heil, On $P^{2}$-irreducible 3-manifolds, Bull. Amer. Math. Soc. 75 (1969), $772-775$.

6. J. M. Kister and D. R. McMillan, Jr., Locally Euclidean factors of $E^{4}$ which cannot be imbedded in $E^{3}$, Ann. of Math. (2) 76 (1962), 541-546.

7. D. R. McMillan, Jr., Some contractible open 3-manifolds, Trans. Amer. Math. Soc. 102 (1962), 373-382.

8. Cartesian products of contractible open 3-manifolds, Bull. Amer. Math. Soc. 67 (1961), 510-514.

9. R. A. Messer, Three dimensional manifolds with finitely generated fundamental groups, Trans. Amer. Math. Soc. 226 (1977), 119-145.

10. J. Stallings, On the loop theorem, Ann. of Math. (2) 72 (1960), 12-19.

11. F. Waldhausen, On irreducible 3-manifolds which are sufficiently large, Ann. of Math. (2) 87 (1968), 56-88.

12. J. H. C. Whitehead, A certain open manifold whose group is unity, Quart. J. Math. Oxford Ser. (2) 6 (1935), 268-279.

13. On 2-spheres in 3-manifolds, Bull. Amer. Math. Soc. 64 (1958), 161-166.

14. J. H. C. Whitehead and M. H. A. Newman, On the group of a certain linkage, Quart. J. Math. Oxford Ser. (2) 8 (1937), 14-22.

Department of Mathematics, Dartmouth College, Hanover, New Hampshire 03755 